\title{
Lithium-Alkyl, -Aryl, and -Amido Ligand Exchange Dynamics of Dianionic Zirconium(IV) Complexes with Chelating Amidophenolate Ligands
}

Karen J. Blackmore, Joseph W. Ziller, and Alan F. Heyduk*

Department of Chemistry, University of California, Irvine, California, 92697

Contents

$\underline{\text { Page }}$

Table S1. ${ }^{1} \mathrm{H}$ and ${ }^{13} \mathrm{C}$ NMR chemical shift data for $\mathbf{3 a}, \mathbf{3 b}, \mathbf{4}$, and $\mathbf{5}$.

Table S2. Crystal data and structure refinement for $\left[\mathrm{Li}\left(\mathrm{OEt}_{2}\right)\right]_{2}\left[\mathrm{Zr}\left(\mathrm{NMe}_{2}\right)_{2}(\mathrm{ap})_{2}\right](\mathbf{5})$.

Table S3. Atomic coordinates and equivalent isotropic displacement parameters for $\left[\mathrm{Li}\left(\mathrm{OEt}_{2}\right)\right]_{2}\left[\mathrm{Zr}\left(\mathrm{NMe}_{2}\right)_{2}(\mathrm{ap})_{2}\right]$ (5).

Table S4. (a) Bond lengths and (b) angles for $\left[\mathrm{Li}\left(\mathrm{OEt}_{2}\right)\right]_{2}\left[\mathrm{Zr}\left(\mathrm{NMe}_{2}\right)_{2}(\mathrm{ap})_{2}\right](\mathbf{5})$.

Table S5. Anisotropic displacement parameters for $\left[\mathrm{Li}\left(\mathrm{OEt}_{2}\right)\right]_{2}\left[\mathrm{Zr}\left(\mathrm{NMe}_{2}\right)_{2}(\mathrm{ap})_{2}\right](\mathbf{5})$.

Table S6. Hydrogen coordinates and isotropic displacement parameters for $\left[\mathrm{Li}\left(\mathrm{OEt}_{2}\right)\right]_{2}\left[\mathrm{Zr}\left(\mathrm{NMe}_{2}\right)_{2}(\mathrm{ap})_{2}\right](\mathbf{5})$.

Figure S1. Sample kinetic plots from ${ }^{1} \mathrm{H}$ NMR data for the reaction of $\mathbf{3 a}$ with $p$ tolyllithium.

Figure S2. Sample kinetic plots from ${ }^{1} \mathrm{H}$ NMR data for the reaction of 4 with $\mathrm{CD}_{3} \mathrm{Li}$. 
Table S1. ${ }^{1} \mathrm{H}$ and ${ }^{13} \mathrm{C}$ NMR chemical shift data for $\mathbf{3 a}, \mathbf{3 b}, \mathbf{4}$, and $\mathbf{5}$.

\begin{tabular}{|c|c|c|c|c|c|c|}
\hline \multirow[b]{2}{*}{$X=$} & \multicolumn{3}{|c|}{${ }^{1} \mathrm{H}$ NMR in THF-d 8} & \multicolumn{3}{|c|}{${ }^{1} \mathrm{H}$ NMR in $\mathrm{C}_{6} \mathrm{D}_{6}$} \\
\hline & $x$ & $\mathrm{C}\left(\mathrm{CH}_{3}\right)_{3}$ & $\mathrm{Ar}$ & $x$ & $\mathrm{C}\left(\mathrm{CH}_{3}\right)_{3}$ & Ar \\
\hline \multirow{3}{*}{$\mathrm{Ph}$} & 6.79 & 1.30 & 6.28 & 6.99 & 1.42 & 6.93 \\
\hline & 6.91 & 1.34 & 6.45 & 7.13 & 1.53 & 7.01 \\
\hline & 8.46 & 1.54 & & 8.78 & 1.78 & \\
\hline \multirow{4}{*}{ tol } & 2.12 & 1.30 & 6.26 & 2.05 & 1.43 & 6.92 \\
\hline & 6.75 & 1.34 & 6.44 & 7.65 & 1.53 & 7.01 \\
\hline & 8.35 & 1.54 & & 6.92 & 1.76 & \\
\hline & & & & 6.93 & & \\
\hline \multirow{3}{*}{$\mathrm{CH}_{3}$} & -0.45 & 1.28 & 6.19 & 0.13 & 1.40 & 6.83 \\
\hline & & 1.33 & 6.40 & & 1.51 & 6.93 \\
\hline & & 1.46 & & & 177.00 & \\
\hline \multirow{3}{*}{$\mathrm{N}\left(\mathrm{CH}_{3}\right)_{2}$} & 2.96 & 1.26 & 6.14 & 3.16 & 1.41 & 6.85 \\
\hline & & 1.42 & 6.46 & & 1.56 & 6.90 \\
\hline & & 1.59 & & & 1.82 & \\
\hline
\end{tabular}

\begin{tabular}{|c|cc|cc|cc|}
\hline \multicolumn{7}{|c|}{${ }^{13} \mathrm{C}$ NMR spectra in THF d } \\
\hline \\
\hline $\mathrm{X}=$ & \multicolumn{2}{|c|}{$\mathrm{X}$} & \multicolumn{2}{c|}{$\mathrm{C}\left(\mathrm{CH}_{3}\right)_{3}$} & \multicolumn{2}{c|}{$\mathrm{Ar}$} \\
\hline \multirow{3}{*}{$\mathrm{Ph}$} & 124.7 & 125.6 & 29.9 & 31.6 & 106.8 & 109.1 \\
& 141.1 & 185.3 & 32.6 & 34.6 & 130.6 & 135.6 \\
& & & 34.7 & 53.5 & 149.7 & 156.0 \\
\hline \multirow{3}{*}{ tol } & 19.9 & & 29.8 & 31.2 & 105.8 & 108.2 \\
& & & 32.7 & 34.6 & 129.8 & 135.7 \\
& & & 34.9 & 53.3 & 149.7 & 156.1 \\
\hline \multirow{3}{*}{$\mathrm{CH}_{3}$} & 21.4 & 126.4 & 29.9 & 31.6 & 106.7 & 109.1 \\
& 132.8 & 141.2 & 32.6 & 34.6 & 130.4 & 135.4 \\
& 181.3 & & 34.8 & 53.5 & 149.8 & 156.2 \\
\hline \multirow{2}{*}{$\mathrm{N}\left(\mathrm{CH}_{3}\right)_{2}$} & 31.1 & & 26.7 & 31.2 & 106.1 & 109.8 \\
& & & 33.2 & 35.3 & 130.2 & 137.0 \\
& & & 35.5 & 54.5 & 152.2 & 154.5 \\
\hline
\end{tabular}


Table S2. Crystal data and structure refinement for $\left[\mathrm{Li}\left(\mathrm{OEt}_{2}\right)\right]_{2}\left[\mathrm{Zr}\left(\mathrm{NMe}_{2}\right)_{2}(\mathrm{ap})_{2}\right](\mathbf{5})$

Empirical formula

Formula weight

Temperature

Wavelength

Crystal system

Space group

Unit cell dimensions

Volume

$\mathrm{Z}$

Density (calculated)

Absorption coefficient

$\mathrm{F}(000)$

Crystal color

Crystal size

Theta range for data collection

Index ranges

Reflections collected

Independent reflections

Completeness to theta $=27.10^{\circ}$

Absorption correction

Max. and min. transmission

Refinement method

Data / restraints / parameters

Goodness-of-fit on $\mathrm{F}^{2}$

Final $R$ indices $[\mathrm{I}>2 \operatorname{sigma}(\mathrm{I})=4640$ data $]$

$\mathrm{R}$ indices (all data; $0.78 \AA$ )

Largest diff. peak and hole
$\mathrm{C}_{48} \mathrm{H}_{90} \mathrm{Li}_{2} \mathrm{~N}_{4} \mathrm{O}_{4} \mathrm{Zr}$

892.34

163(2) K

$0.71073 \AA$

Orthorhombic

Pbcn

$\mathrm{a}=17.682(2) \AA \quad \alpha=90^{\circ}$.

$\mathrm{b}=17.923(2) \AA \quad \beta=90^{\circ}$.

$\mathrm{c}=16.4037(19) \AA \quad \gamma=90^{\circ}$.

5198.6(10) $\AA^{3}$

4

$1.140 \mathrm{Mg} / \mathrm{m}^{3}$

$0.253 \mathrm{~mm}^{-1}$

1936

yellow

$0.42 \times 0.24 \times 0.20 \mathrm{~mm}^{3}$

1.62 to $27.10^{\circ}$

$-22 \leq h \leq 22,-22 \leq k \leq 22,-21 \leq l \leq 21$

51186

$5729[\mathrm{R}($ int $)=0.0374]$

$100.0 \%$

Semi-empirical from equivalents

0.9511 and 0.9011

Full-matrix least-squares on $\mathrm{F}^{2}$

5729 / 0 / 265

1.063

$\mathrm{R} 1=0.0486, \mathrm{wR} 2=0.1191$

$\mathrm{R} 1=0.0622, \mathrm{wR} 2=0.1298$

1.325 and -0.607 e. $\AA^{-3}$ 
Table S3. Atomic coordinates $\left(\times 10^{4}\right)$ and equivalent isotropic displacement parameters $\left(\AA^{2}\right.$ x $\left.10^{3}\right)$ for $\left[\mathrm{Li}\left(\mathrm{OEt}_{2}\right)\right]_{2}\left[\mathrm{Zr}\left(\mathrm{NMe}_{2}\right)_{2}(\mathrm{ap})_{2}\right](\mathbf{5})$. U(eq) is defined as one third of the trace of the orthogonalized $\mathrm{U}^{\mathrm{ij}}$ tensor.

\begin{tabular}{|c|c|c|c|c|}
\hline & $\mathbf{x}$ & $y$ & $\mathbf{z}$ & $\mathrm{U}(\mathrm{eq})$ \\
\hline $\operatorname{Zr}(1)$ & 0 & 1901(1) & 7500 & $23(1)$ \\
\hline $\mathrm{Li}(1)$ & $1281(4)$ & $1747(4)$ & $6400(4)$ & $62(2)$ \\
\hline $\mathrm{O}(1)$ & $-242(1)$ & $1815(1)$ & $6259(1)$ & $25(1)$ \\
\hline $\mathrm{O}(2)$ & 1958(2) & $1415(2)$ & $5558(2)$ & $74(1)$ \\
\hline$N(1)$ & $720(1)$ & $2752(1)$ & $6813(1)$ & $22(1)$ \\
\hline $\mathrm{N}(2)$ & $923(2)$ & $1087(1)$ & $7341(1)$ & $36(1)$ \\
\hline$C(1)$ & $565(1)$ & $2819(1)$ & $5972(1)$ & $22(1)$ \\
\hline$C(2)$ & $867(2)$ & $3322(2)$ & $5405(2)$ & $27(1)$ \\
\hline$C(3)$ & $652(2)$ & $3312(2)$ & $4587(2)$ & $32(1)$ \\
\hline$C(4)$ & $141(2)$ & 2773(2) & $4330(2)$ & $30(1)$ \\
\hline$C(5)$ & $-172(1)$ & $2243(1)$ & $4855(2)$ & $24(1)$ \\
\hline$C(6)$ & $38(1)$ & $2280(1)$ & $5683(1)$ & $22(1)$ \\
\hline$C(7)$ & $1270(1)$ & $3279(1)$ & $7192(2)$ & $24(1)$ \\
\hline $\mathrm{C}(8)$ & 1053(2) & $4104(2)$ & $7088(2)$ & $40(1)$ \\
\hline $\mathrm{C}(9)$ & $2077(2)$ & $3132(2)$ & 6882(2) & $42(1)$ \\
\hline$C(10)$ & $1275(2)$ & $3130(2)$ & $8111(2)$ & $36(1)$ \\
\hline$C(11)$ & $956(2)$ & $3892(2)$ & $3979(2)$ & $49(1)$ \\
\hline$C(12)$ & $1338(4)$ & $3463(4)$ & $3253(3)$ & $114(2)$ \\
\hline$C(13)$ & $338(3)$ & $4313(4)$ & $3627(4)$ & $143(4)$ \\
\hline$C(14)$ & $1575(3)$ & $4364(3)$ & $4308(3)$ & $83(2)$ \\
\hline$C(15)$ & $-738(2)$ & $1657(2)$ & $4547(2)$ & $27(1)$ \\
\hline$C(16)$ & $-1517(2)$ & 1812(2) & $4912(2)$ & $35(1)$ \\
\hline$C(17)$ & $-817(2)$ & $1670(2)$ & $3611(2)$ & $35(1)$ \\
\hline$C(18)$ & $-483(2)$ & $866(2)$ & $4788(2)$ & $37(1)$ \\
\hline$C(19)$ & $769(3)$ & $403(2)$ & $6887(3)$ & $78(2)$ \\
\hline$C(20)$ & $1474(2)$ & $897(2)$ & $7966(2)$ & $48(1)$ \\
\hline$C(21)$ & $1873(2)$ & 1653(2) & $4734(2)$ & $52(1)$ \\
\hline$C(22)$ & $1718(2)$ & $1032(2)$ & $4140(3)$ & $66(1)$ \\
\hline$C(23)$ & $2467(4)$ & $781(4)$ & $5800(4)$ & $51(2)$ \\
\hline$C(24)$ & $3250(4)$ & $1100(4)$ & $5784(5)$ & $64(2)$ \\
\hline$C(23 B)$ & $2716(5)$ & $1073(6)$ & $5533(6)$ & $59(2)$ \\
\hline$C(24 B)$ & $3028(6)$ & 1104(6) & $6321(6)$ & $66(2)$ \\
\hline
\end{tabular}


Table S4. (a) Bond lengths $[\AA ̊ \mathrm{~A}]$ for $\left[\mathrm{Li}\left(\mathrm{OEt}_{2}\right)\right]_{2}\left[\mathrm{Zr}\left(\mathrm{NMe}_{2}\right)_{2}(\mathrm{ap})_{2}\right]$ (5).

\begin{tabular}{|lcll|}
\hline $\mathrm{Zr}(1)-\mathrm{O}(1)$ & $2.0863(17)$ & $\mathrm{N}(2)-\mathrm{C}(19)$ & $1.461(4)$ \\
$\mathrm{Zr}(1)-\mathrm{O}(1) \# 1$ & $2.0863(17)$ & $\mathrm{C}(1)-\mathrm{C}(2)$ & $1.401(4)$ \\
$\mathrm{Zr}(1)-\mathrm{N}(2)$ & $2.204(2)$ & $\mathrm{C}(1)-\mathrm{C}(6)$ & $1.424(3)$ \\
$\mathrm{Zr}(1)-\mathrm{N}(2) \# 1$ & $2.204(2)$ & $\mathrm{C}(2)-\mathrm{C}(3)$ & $1.394(4)$ \\
$\mathrm{Zr}(1)-\mathrm{N}(1) \# 1$ & $2.285(2)$ & $\mathrm{C}(3)-\mathrm{C}(4)$ & $1.390(4)$ \\
$\mathrm{Zr}(1)-\mathrm{N}(1)$ & $\mathrm{C}(3)-\mathrm{C}(11)$ & $1.537(4)$ \\
$\mathrm{Zr}(1)-\mathrm{Li}(1) \# 1$ & $2.285(2)$ & $\mathrm{C}(4)-\mathrm{C}(5)$ & $1.398(4)$ \\
$\mathrm{Zr}(1)-\mathrm{Li}(1)$ & $\mathrm{C}(5)-\mathrm{C}(6)$ & $1.410(3)$ \\
$\mathrm{Li}(1)-\mathrm{O}(2)$ & $\mathrm{C}(5)-\mathrm{C}(15)$ & $1.536(3)$ \\
$\mathrm{Li}(1)-\mathrm{N}(2)$ & $\mathrm{C}(7)-\mathrm{C}(10)$ & $1.530(4)$ \\
$\mathrm{Li}(1)-\mathrm{N}(1)$ & $\mathrm{C}(7)-\mathrm{C}(8)$ & $1.537(4)$ \\
$\mathrm{Li}(1)-\mathrm{C}(1)$ & $\mathrm{C}(7)-\mathrm{C}(9)$ & $1.538(4)$ \\
$\mathrm{Li}(1)-\mathrm{C}(6)$ & $1.922(6)$ & $\mathrm{C}(11)-\mathrm{C}(13)$ & $1.448(6)$ \\
$\mathrm{Li}(1)-\mathrm{C}(19)$ & $2.045(7)$ & $\mathrm{C}(11)-\mathrm{C}(14)$ & $1.485(5)$ \\
$\mathrm{O}(1)-\mathrm{C}(6)$ & $2.166(6)$ & $\mathrm{C}(11)-\mathrm{C}(12)$ & $1.572(6)$ \\
$\mathrm{O}(2)-\mathrm{C}(21)$ & $2.406(6)$ & $\mathrm{C}(15)-\mathrm{C}(16)$ & $1.527(4)$ \\
$\mathrm{O}(2)-\mathrm{C}(23 \mathrm{~B})$ & $2.668(7)$ & $\mathrm{C}(15)-\mathrm{C}(18)$ & $1.539(4)$ \\
$\mathrm{O}(2)-\mathrm{C}(23)$ & $2.693(9)$ & $\mathrm{C}(15)-\mathrm{C}(17)$ & $1.543(3)$ \\
$\mathrm{N}(1)-\mathrm{C}(1)$ & $1.354(3)$ & $\mathrm{C}(21)-\mathrm{C}(22)$ & $1.504(5)$ \\
$\mathrm{N}(1)-\mathrm{C}(7)$ & $1.425(5)$ & $\mathrm{C}(23)-\mathrm{C}(24)$ & $1.498(10)$ \\
$\mathrm{N}(2)-\mathrm{C}(20)$ & $1.475(9)$ & $\mathrm{C}(23 \mathrm{~B})-\mathrm{C}(24 \mathrm{~B})$ & $1.407(13)$ \\
\hline
\end{tabular}

Table S4. (b) Bond angles $[\mathrm{deg}]$ for $\left[\mathrm{Li}\left(\mathrm{OEt}_{2}\right)\right]_{2}\left[\mathrm{Zr}\left(\mathrm{NMe}_{2}\right)_{2}(\mathrm{ap})_{2}\right](5)$.

\begin{tabular}{|c|c|c|c|}
\hline $\mathrm{O}(1)-\mathrm{Zr}(1)-\mathrm{O}(1) \# 1$ & $171.53(9)$ & $\mathrm{C}(23)-\mathrm{O}(2)-\mathrm{Li}(1)$ & $114.7(4)$ \\
\hline$O(1)-\operatorname{Zr}(1)-N(2)$ & $89.31(7)$ & $\mathrm{C}(1)-\mathrm{N}(1)-\mathrm{C}(7)$ & $118.7(2)$ \\
\hline $\mathrm{O}(1) \# 1-\mathrm{Zr}(1)-\mathrm{N}(2)$ & $85.09(7)$ & $\mathrm{C}(1)-\mathrm{N}(1)-\mathrm{Li}(1)$ & $81.6(2)$ \\
\hline $\mathrm{O}(1)-\mathrm{Zr}(1)-\mathrm{N}(2) \# 1$ & $85.09(7)$ & $\mathrm{C}(7)-\mathrm{N}(1)-\mathrm{Li}(1)$ & $111.0(3)$ \\
\hline $\mathrm{O}(1) \# 1-\operatorname{Zr}(1)-\mathrm{N}(2) \# 1$ & $89.31(8)$ & $C(1)-N(1)-Z r(1)$ & $115.56(15)$ \\
\hline$N(2)-Z r(1)-N(2) \# 1$ & $97.14(14)$ & $\mathrm{C}(7)-\mathrm{N}(1)-\mathrm{Zr}(1)$ & $125.45(15)$ \\
\hline $\mathrm{O}(1)-\mathrm{Zr}(1)-\mathrm{N}(1) \# 1$ & $114.63(7)$ & $\mathrm{Li}(1)-\mathrm{N}(1)-\mathrm{Zr}(1)$ & $81.6(2)$ \\
\hline $\mathrm{O}(1) \# 1-\operatorname{Zr}(1)-\mathrm{N}(1) \# 1$ & $71.45(7)$ & $\mathrm{C}(20)-\mathrm{N}(2)-\mathrm{C}(19)$ & 106.7(3) \\
\hline$N(2)-Z r(1)-N(1) \# 1$ & $155.87(7)$ & $C(20)-N(2)-\operatorname{Li}(1)$ & $117.4(3)$ \\
\hline$N(2) \# 1-\operatorname{Zr}(1)-N(1) \# 1$ & $88.35(9)$ & $C(19)-N(2)-\operatorname{Li}(1)$ & $99.0(3)$ \\
\hline$O(1)-\operatorname{Zr}(1)-N(1)$ & $71.46(7)$ & $\mathrm{C}(20)-\mathrm{N}(2)-\mathrm{Zr}(1)$ & $124.63(18)$ \\
\hline $\mathrm{O}(1) \# 1-\mathrm{Zr}(1)-\mathrm{N}(1)$ & $114.63(7)$ & $\mathrm{C}(19)-\mathrm{N}(2)-\mathrm{Zr}(1)$ & $118.5(2)$ \\
\hline$N(2)-\operatorname{Zr}(1)-N(1)$ & $88.35(9)$ & $\mathrm{Li}(1)-\mathrm{N}(2)-\mathrm{Zr}(1)$ & $86.32(18)$ \\
\hline$N(2) \# 1-Z r(1)-N(1)$ & $155.88(7)$ & $C(2)-C(1)-N(1)$ & $129.0(2)$ \\
\hline$N(1) \# 1-Z r(1)-N(1)$ & $96.19(10)$ & $C(2)-C(1)-C(6)$ & $117.7(2)$ \\
\hline $\mathrm{O}(1)-\mathrm{Zr}(1)-\mathrm{Li}(1) \# 1$ & $116.03(14)$ & $N(1)-C(1)-C(6)$ & 113.2(2) \\
\hline
\end{tabular}




\begin{tabular}{|c|c|c|c|}
\hline $\mathrm{O}(1) \# 1-\mathrm{Zr}(1)-\mathrm{Li}(1) \# 1$ & $63.07(15)$ & $C(2)-C(1)-L i(1)$ & $120.5(2)$ \\
\hline$N(2)-Z r(1)-\operatorname{Li}(1) \# 1$ & $125.92(16)$ & $N(1)-C(1)-L i(1)$ & $62.95(18)$ \\
\hline$N(2) \# 1-\operatorname{Zr}(1)-\operatorname{Li}(1) \# 1$ & $44.56(13)$ & $C(6)-C(1)-L i(1)$ & $84.2(2)$ \\
\hline$N(1) \# 1-\operatorname{Zr}(1)-\operatorname{Li}(1) \# 1$ & $47.44(14)$ & $C(3)-C(2)-C(1)$ & $121.8(2)$ \\
\hline$N(1)-\operatorname{Zr}(1)-\operatorname{Li}(1) \# 1$ & $143.45(14)$ & $C(4)-C(3)-C(2)$ & 118.6(2) \\
\hline$O(1)-\operatorname{Zr}(1)-\operatorname{Li}(1)$ & $63.07(15)$ & $C(4)-C(3)-C(11)$ & $120.0(2)$ \\
\hline $\mathrm{O}(1) \# 1-\mathrm{Zr}(1)-\mathrm{Li}(1)$ & $116.03(14)$ & $C(2)-C(3)-C(11)$ & $121.4(2)$ \\
\hline$N(2)-\operatorname{Zr}(1)-\operatorname{Li}(1)$ & $44.56(13)$ & $C(3)-C(4)-C(5)$ & $122.9(2)$ \\
\hline$N(2) \# 1-Z r(1)-\operatorname{Li}(1)$ & $125.92(16)$ & $C(4)-C(5)-C(6)$ & $117.2(2)$ \\
\hline$N(1) \# 1-\operatorname{Zr}(1)-\operatorname{Li}(1)$ & $143.45(14)$ & $C(4)-C(5)-C(15)$ & $121.4(2)$ \\
\hline$N(1)-\operatorname{Zr}(1)-\operatorname{Li}(1)$ & $47.44(14)$ & $C(6)-C(5)-C(15)$ & $121.4(2)$ \\
\hline Li(1)\#1-Zr(1)-Li(1) & $169.1(3)$ & $\mathrm{O}(1)-\mathrm{C}(6)-\mathrm{C}(5)$ & $123.1(2)$ \\
\hline $\mathrm{O}(2)-\mathrm{Li}(1)-\mathrm{N}(2)$ & $123.8(3)$ & $\mathrm{O}(1)-\mathrm{C}(6)-\mathrm{C}(1)$ & $115.2(2)$ \\
\hline $\mathrm{O}(2)-\mathrm{Li}(1)-\mathrm{N}(1)$ & $140.1(4)$ & $C(5)-C(6)-C(1)$ & $121.7(2)$ \\
\hline$N(2)-\mathrm{Li}(1)-\mathrm{N}(1)$ & $95.9(3)$ & $\mathrm{O}(1)-\mathrm{C}(6)-\mathrm{Li}(1)$ & $76.9(2)$ \\
\hline $\mathrm{O}(2)-\mathrm{Li}(1)-\mathrm{C}(1)$ & $111.4(3)$ & $C(5)-C(6)-L i(1)$ & $128.7(2)$ \\
\hline$N(2)-L i(1)-C(1)$ & 121.3(3) & $C(1)-C(6)-L i(1)$ & $63.8(2)$ \\
\hline$N(1)-L i(1)-C(1)$ & $35.46(11)$ & $N(1)-C(7)-C(10)$ & $107.7(2)$ \\
\hline $\mathrm{O}(2)-\mathrm{Li}(1)-\mathrm{C}(6)$ & $107.9(3)$ & $N(1)-C(7)-C(8)$ & $113.5(2)$ \\
\hline$N(2)-\mathrm{Li}(1)-\mathrm{C}(6)$ & 106.6(3) & $C(10)-C(7)-C(8)$ & $106.2(2)$ \\
\hline$N(1)-\operatorname{Li}(1)-C(6)$ & $57.50(16)$ & $N(1)-C(7)-C(9)$ & $111.0(2)$ \\
\hline$C(1)-\mathrm{Li}(1)-C(6)$ & $32.06(11)$ & $C(10)-C(7)-C(9)$ & $106.9(2)$ \\
\hline $\mathrm{O}(2)-\mathrm{Li}(1)-\mathrm{C}(19)$ & $98.4(3)$ & $C(8)-C(7)-C(9)$ & $111.1(2)$ \\
\hline$N(2)-\operatorname{Li}(1)-C(19)$ & $32.39(16)$ & $C(13)-C(11)-C(14)$ & $113.9(4)$ \\
\hline$N(1)-\operatorname{Li}(1)-C(19)$ & $119.8(3)$ & $C(13)-C(11)-C(3)$ & $110.4(3)$ \\
\hline$C(1)-\operatorname{Li}(1)-C(19)$ & 128.6(3) & $C(14)-C(11)-C(3)$ & $114.0(3)$ \\
\hline$C(6)-\operatorname{Li}(1)-C(19)$ & $100.1(3)$ & $C(13)-C(11)-C(12)$ & $106.1(5)$ \\
\hline $\mathrm{O}(2)-\mathrm{Li}(1)-\mathrm{Zr}(1)$ & $163.8(4)$ & $C(14)-C(11)-C(12)$ & $103.7(4)$ \\
\hline$N(2)-\operatorname{Li}(1)-\operatorname{Zr}(1)$ & $49.12(16)$ & $C(3)-C(11)-C(12)$ & $108.1(3)$ \\
\hline$N(1)-\operatorname{Li}(1)-\operatorname{Zr}(1)$ & $50.98(14)$ & $C(16)-C(15)-C(5)$ & $109.5(2)$ \\
\hline$C(1)-\operatorname{Li}(1)-\operatorname{Zr}(1)$ & $72.29(17)$ & $C(16)-C(15)-C(18)$ & $109.4(2)$ \\
\hline$C(6)-\operatorname{Li}(1)-\operatorname{Zr}(1)$ & $66.33(17)$ & $C(5)-C(15)-C(18)$ & $110.8(2)$ \\
\hline$C(19)-\operatorname{Li}(1)-\operatorname{Zr}(1)$ & $68.9(2)$ & $C(16)-C(15)-C(17)$ & $107.8(2)$ \\
\hline$C(6)-O(1)-Z r(1)$ & $124.07(14)$ & $C(5)-C(15)-C(17)$ & $112.0(2)$ \\
\hline $\mathrm{C}(21)-\mathrm{O}(2)-\mathrm{C}(23 \mathrm{~B})$ & $101.1(4)$ & $C(18)-C(15)-C(17)$ & 107.2(2) \\
\hline$C(21)-O(2)-C(23)$ & $122.7(4)$ & $N(2)-C(19)-\operatorname{Li}(1)$ & $48.6(2)$ \\
\hline $\mathrm{C}(23 \mathrm{~B})-\mathrm{O}(2)-\mathrm{C}(23)$ & $31.6(4)$ & $\mathrm{O}(2)-\mathrm{C}(21)-\mathrm{C}(22)$ & $114.3(3)$ \\
\hline $\mathrm{C}(21)-\mathrm{O}(2)-\mathrm{Li}(1)$ & $121.5(3)$ & $\mathrm{C}(24)-\mathrm{C}(23)-\mathrm{O}(2)$ & $105.1(6)$ \\
\hline $\mathrm{C}(23 \mathrm{~B})-\mathrm{O}(2)-\mathrm{Li}(1)$ & $135.7(5)$ & $C(24 B)-C(23 B)-O(2)$ & $108.3(8)$ \\
\hline
\end{tabular}

Symmetry transformations used to generate equivalent atoms: \#1 -x,y,-z+3/2 
Table S5. Anisotropic displacement parameters $\left(\AA^{2} \times 10^{3}\right)$ for $\left[\mathrm{Li}\left(\mathrm{OEt}_{2}\right)\right]_{2}\left[\mathrm{Zr}\left(\mathrm{NMe}_{2}\right)_{2}(\mathrm{ap})_{2}\right](\mathbf{5})$. The anisotropic displacement factor exponent takes the form: $-2 \pi^{2}\left[h^{2} a^{* 2} U^{11}+\ldots+2 h k a^{*} b^{*} U^{12}\right]$.

\begin{tabular}{|c|c|c|c|c|c|c|}
\hline & U11 & $\mathbf{U} 22$ & U33 & $\mathbf{U} 23$ & U13 & U12 \\
\hline $\operatorname{Zr}(1)$ & $30(1)$ & $19(1)$ & $19(1)$ & 0 & $-7(1)$ & 0 \\
\hline $\operatorname{Li}(1)$ & $99(5)$ & $52(3)$ & $36(3)$ & $1(3)$ & $9(3)$ & $45(4)$ \\
\hline $\mathrm{O}(1)$ & $31(1)$ & $24(1)$ & $19(1)$ & $-1(1)$ & $-5(1)$ & $-5(1)$ \\
\hline $\mathrm{O}(2)$ & $81(2)$ & $90(2)$ & $50(2)$ & $-22(1)$ & $-4(1)$ & $54(2)$ \\
\hline $\mathrm{N}(1)$ & $23(1)$ & $23(1)$ & $20(1)$ & $-4(1)$ & $-5(1)$ & $1(1)$ \\
\hline $\mathrm{N}(2)$ & $52(2)$ & $27(1)$ & $27(1)$ & $-7(1)$ & $-15(1)$ & $12(1)$ \\
\hline $\mathrm{C}(1)$ & $22(1)$ & $23(1)$ & $21(1)$ & $-3(1)$ & $-3(1)$ & $2(1)$ \\
\hline $\mathrm{C}(2)$ & $26(1)$ & $29(1)$ & $27(1)$ & $-1(1)$ & $-3(1)$ & $-6(1)$ \\
\hline$C(3)$ & $34(1)$ & $37(1)$ & $26(1)$ & $5(1)$ & $-2(1)$ & $-9(1)$ \\
\hline C(4) & $31(1)$ & $38(2)$ & $20(1)$ & $1(1)$ & $-4(1)$ & $-4(1)$ \\
\hline$C(5)$ & $23(1)$ & $27(1)$ & $21(1)$ & $-2(1)$ & $-4(1)$ & $0(1)$ \\
\hline$C(6)$ & $25(1)$ & $21(1)$ & $21(1)$ & $0(1)$ & $-1(1)$ & $1(1)$ \\
\hline$C(7)$ & $22(1)$ & $29(1)$ & $22(1)$ & $-7(1)$ & $0(1)$ & $-5(1)$ \\
\hline $\mathrm{C}(8)$ & $48(2)$ & $28(1)$ & $46(2)$ & $-10(1)$ & $-2(1)$ & $-7(1)$ \\
\hline C(9) & $24(1)$ & $68(2)$ & $33(2)$ & $-13(2)$ & $0(1)$ & $-3(1)$ \\
\hline$C(10)$ & $37(2)$ & $48(2)$ & $23(1)$ & $-4(1)$ & $-5(1)$ & $-15(1)$ \\
\hline $\mathrm{C}(11)$ & $49(2)$ & $66(2)$ & $31(2)$ & $19(2)$ & $-8(1)$ & $-27(2)$ \\
\hline $\mathrm{C}(12)$ & $145(6)$ & $127(5)$ & $69(3)$ & $-7(3)$ & $50(3)$ & $-65(4)$ \\
\hline$C(13)$ & $79(3)$ & $160(6)$ & 190(7) & $150(6)$ & $-67(4)$ & $-62(4)$ \\
\hline$C(14)$ & $73(3)$ & $101(4)$ & $76(3)$ & $54(3)$ & $-27(2)$ & $-51(3)$ \\
\hline$C(15)$ & $33(1)$ & $29(1)$ & $20(1)$ & $-1(1)$ & $-6(1)$ & $-4(1)$ \\
\hline$C(16)$ & $31(1)$ & $46(2)$ & $28(1)$ & $-2(1)$ & $-5(1)$ & $-7(1)$ \\
\hline$C(17)$ & $39(2)$ & $41(2)$ & $24(1)$ & $-4(1)$ & $-8(1)$ & $-7(1)$ \\
\hline $\mathrm{C}(18)$ & $49(2)$ & $29(1)$ & $31(1)$ & $-4(1)$ & $-12(1)$ & $-4(1)$ \\
\hline$C(19)$ & $85(3)$ & $45(2)$ & $103(3)$ & $-39(2)$ & $-50(3)$ & $27(2)$ \\
\hline $\mathrm{C}(20)$ & $54(2)$ & $57(2)$ & $32(2)$ & $-7(1)$ & $-10(1)$ & $27(2)$ \\
\hline $\mathrm{C}(21)$ & $48(2)$ & $60(2)$ & $48(2)$ & $-19(2)$ & $13(2)$ & $0(2)$ \\
\hline $\mathrm{C}(22)$ & $61(2)$ & $77(3)$ & $60(2)$ & $-25(2)$ & $8(2)$ & $-13(2)$ \\
\hline
\end{tabular}


Table S6. Hydrogen coordinates ( $\left.\times 10^{4}\right)$ and isotropic displacement parameters $\left(\AA^{2} \times 10^{3}\right)$ for $\left[\mathrm{Li}\left(\mathrm{OEt}_{2}\right)\right]_{2}\left[\mathrm{Zr}\left(\mathrm{NMe}_{2}\right)_{2}(\mathrm{ap})_{2}\right](\mathbf{5})$.

\begin{tabular}{|c|c|c|c|c|}
\hline & $x$ & $y$ & $z$ & $\mathrm{U}(\mathrm{eq})$ \\
\hline $\mathrm{H}(2 \mathrm{~A})$ & 1228 & 3680 & 5582 & 33 \\
\hline $\mathrm{H}(4 \mathrm{~A})$ & -1 & 2763 & 3771 & 36 \\
\hline$H(8 A)$ & 537 & 4182 & 7289 & 61 \\
\hline $\mathrm{H}(8 \mathrm{~B})$ & 1078 & 4238 & 6510 & 61 \\
\hline $\mathrm{H}(8 \mathrm{C})$ & 1405 & 4416 & 7399 & 61 \\
\hline $\mathrm{H}(9 \mathrm{~A})$ & 2098 & 3222 & 6293 & 63 \\
\hline $\mathrm{H}(9 \mathrm{~B})$ & 2216 & 2612 & 6994 & 63 \\
\hline $\mathrm{H}(9 \mathrm{C})$ & 2431 & 3466 & 7160 & 63 \\
\hline$H(10 A)$ & 768 & 3218 & 8333 & 54 \\
\hline $\mathrm{H}(10 \mathrm{~B})$ & 1637 & 3464 & 8376 & 54 \\
\hline $\mathrm{H}(10 \mathrm{C})$ & 1422 & 2610 & 8211 & 54 \\
\hline$H(12 A)$ & 1536 & 3823 & 2857 & 170 \\
\hline $\mathrm{H}(12 \mathrm{~B})$ & 963 & 3142 & 2987 & 170 \\
\hline$H(12 C)$ & 1754 & 3156 & 3461 & 170 \\
\hline$H(13 A)$ & 78 & 4593 & 4057 & 215 \\
\hline$H(13 B)$ & -19 & 3970 & 3366 & 215 \\
\hline$H(13 C)$ & 537 & 4661 & 3219 & 215 \\
\hline$H(14 A)$ & 1387 & 4655 & 4771 & 125 \\
\hline$H(14 B)$ & 1755 & 4704 & 3882 & 125 \\
\hline$H(14 C)$ & 1993 & 4045 & 4488 & 125 \\
\hline$H(16 A)$ & -1483 & 1806 & 5508 & 52 \\
\hline$H(16 B)$ & -1874 & 1428 & 4731 & 52 \\
\hline$H(16 C)$ & -1694 & 2303 & 4730 & 52 \\
\hline$H(17 A)$ & -981 & 2168 & 3435 & 52 \\
\hline $\mathrm{H}(17 \mathrm{~B})$ & -1192 & 1299 & 3440 & 52 \\
\hline$H(17 C)$ & -328 & 1553 & 3361 & 52 \\
\hline $\mathrm{H}(18 \mathrm{~A})$ & -428 & 836 & 5381 & 55 \\
\hline $\mathrm{H}(18 \mathrm{~B})$ & 4 & 755 & 4528 & 55 \\
\hline $\mathrm{H}(18 \mathrm{C})$ & -862 & 502 & 4606 & 55 \\
\hline$H(19 A)$ & 1228 & 97 & 6865 & 117 \\
\hline $\mathrm{H}(19 \mathrm{~B})$ & 610 & 530 & 6331 & 117 \\
\hline$H(19 C)$ & 365 & 123 & 7158 & 117 \\
\hline $\mathrm{H}(20 \mathrm{~A})$ & 1826 & 523 & 7752 & 72 \\
\hline $\mathrm{H}(20 \mathrm{~B})$ & 1212 & 694 & 8444 & 72 \\
\hline $\mathrm{H}(20 \mathrm{C})$ & 1756 & 1345 & 8123 & 72 \\
\hline $\mathrm{H}(21 \mathrm{~A})$ & 2341 & 1914 & 4565 & 62 \\
\hline
\end{tabular}




\begin{tabular}{|lcccc|}
\hline$H(21 B)$ & 1453 & 2017 & 4706 & 62 \\
$H(22 A)$ & 1669 & 1239 & 3590 & 99 \\
$H(22 B)$ & 1248 & 779 & 4291 & 99 \\
$H(22 C)$ & 2137 & 674 & 4152 & 99 \\
$H(23 A)$ & 2339 & 599 & 6353 & 61 \\
$H(23 B)$ & 2421 & 362 & 5410 & 61 \\
$H(24 A)$ & 3614 & 713 & 5938 & 96 \\
$H(24 B)$ & 3282 & 1516 & 6170 & 96 \\
$H(24 C)$ & 3365 & 1280 & 5233 & 96 \\
$H(23 C)$ & 2679 & 548 & 5350 & 70 \\
$H(23 D)$ & 3043 & 1347 & 5145 & 70 \\
$H(24 D)$ & 3535 & 885 & 6315 & 99 \\
$H(24 E)$ & 2706 & 824 & 6699 & 99 \\
$H(24 F)$ & 3059 & 1625 & 6499 & 99 \\
\hline
\end{tabular}


Figure S1. Sample kinetic plots from ${ }^{1} \mathrm{H}$ NMR data for the reaction of $\mathbf{3 a}$ with $p$-tolyllithium.
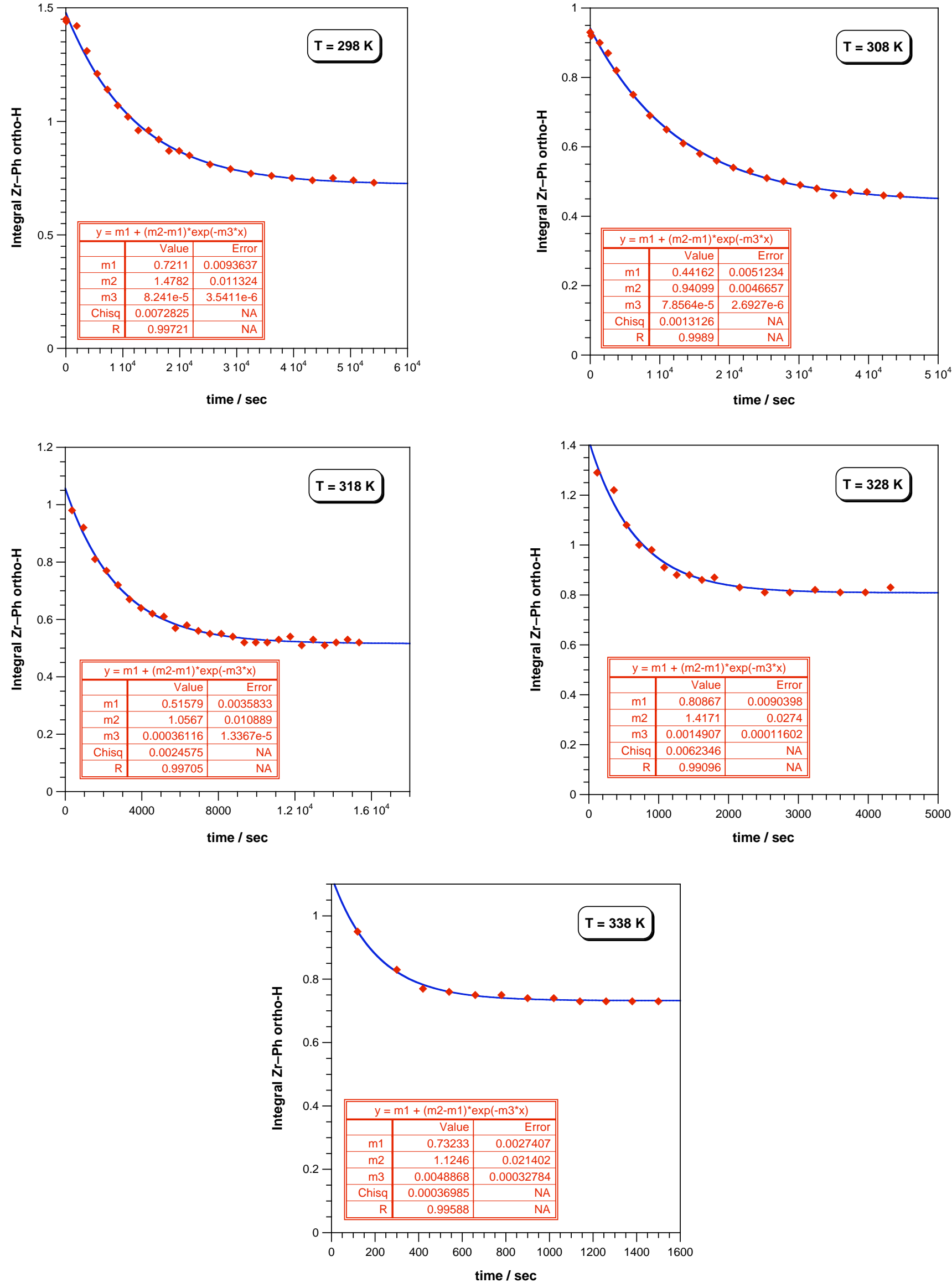
Figure S2. Sample kinetic plots from ${ }^{1} \mathrm{H}$ NMR data for the reaction of $\mathbf{4}$ with $\mathrm{CD}_{3} \mathrm{Li}$.
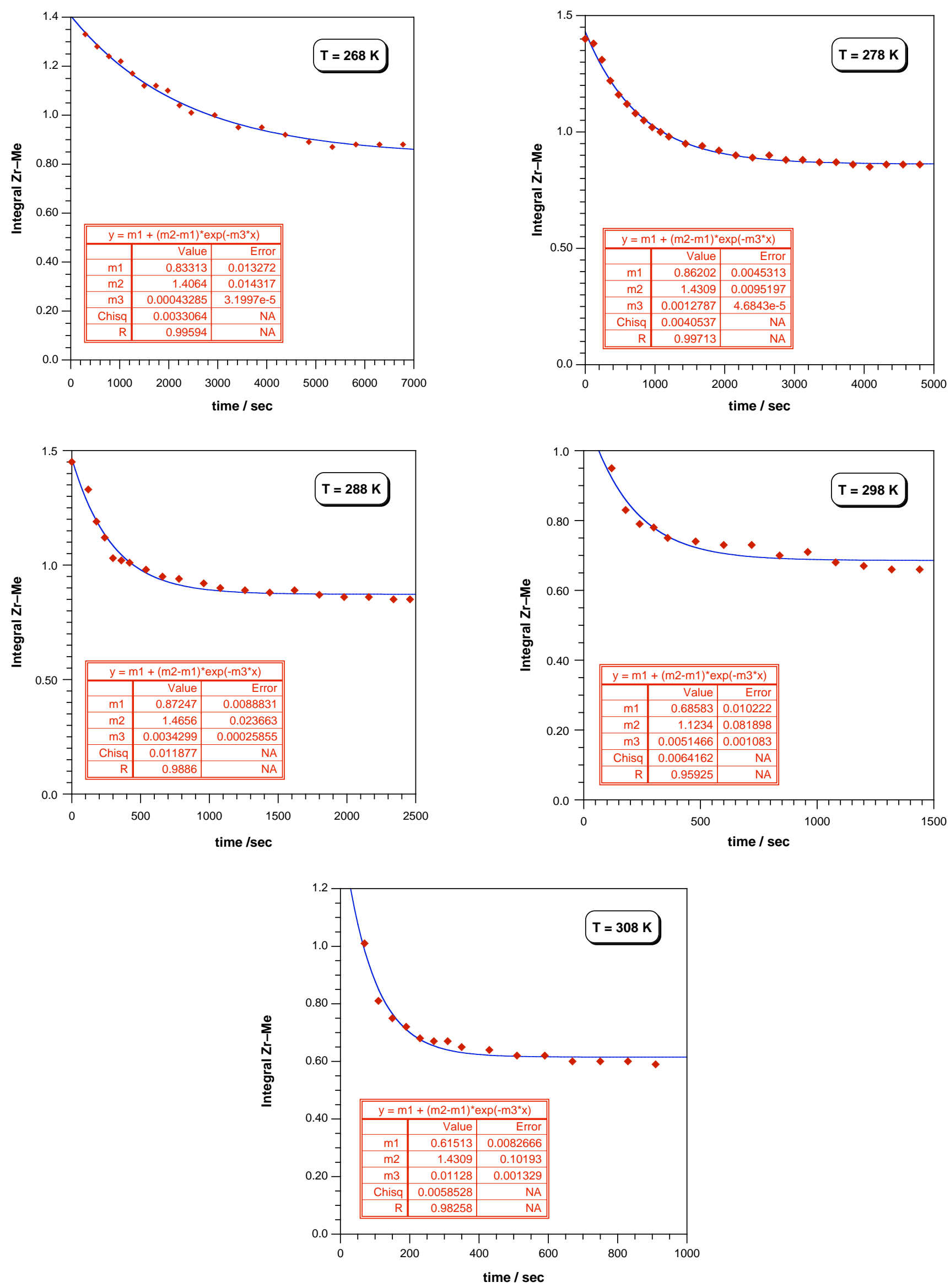
Figure S3. Variable temperature ${ }^{1} \mathrm{H}$ NMR data for the reaction of 5 with $\mathrm{LiNMe}_{2}$.

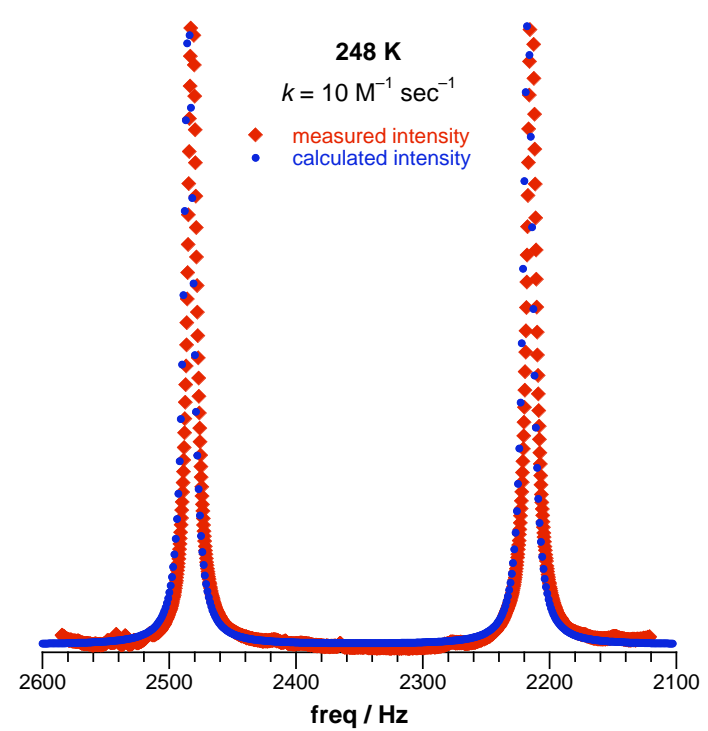

$268 \mathrm{~K}$

$k=250 \mathrm{M}^{-1} \sec ^{-1}$

- measured intensity

- calculated intensity

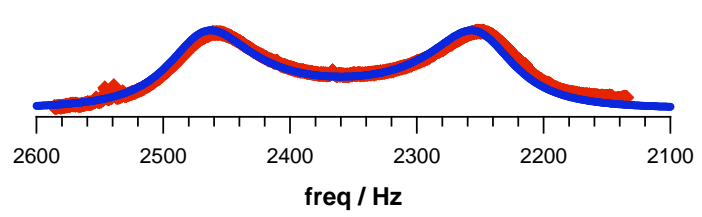

$288 \mathrm{~K}$

$k=3400 \mathrm{M}^{-1} \mathrm{sec}^{-1}$

- measured intensity

- calculated intensity

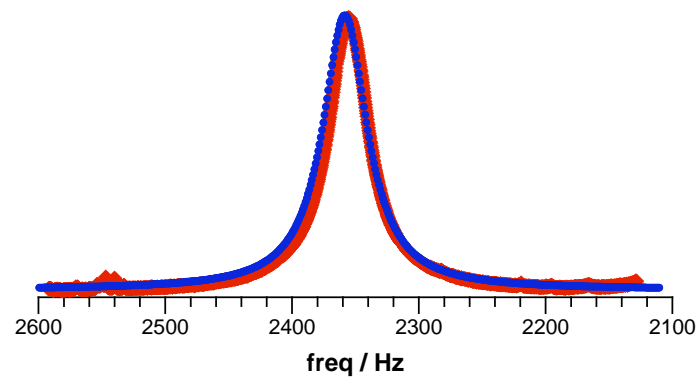

$258 \mathrm{~K}$

$k=53 \mathrm{M}^{-1} \mathrm{sec}^{-1}$

- $\quad$ measured intensity

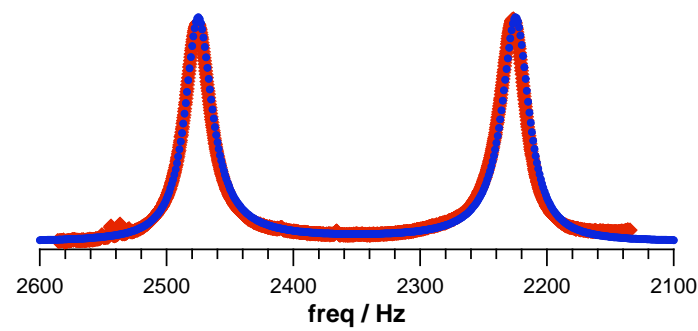

$278 \mathrm{~K}$

$k=1150 \mathrm{M}^{-1} \mathrm{sec}^{-1}$

- $\quad$ measured intensity

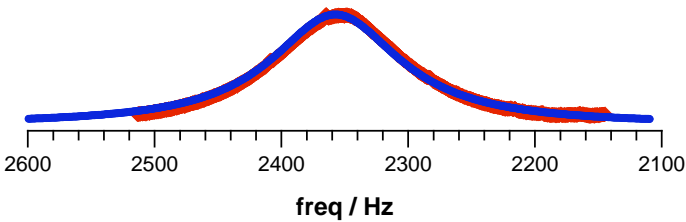

$298 \mathrm{~K}$

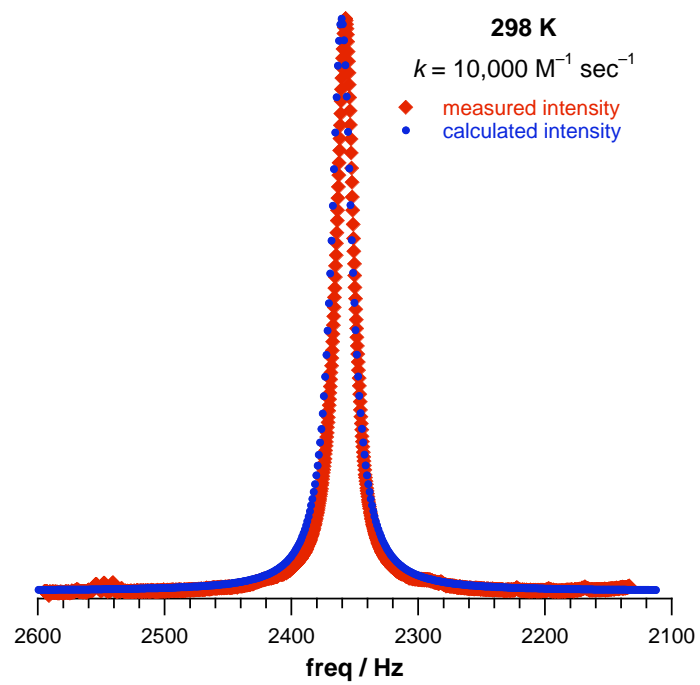

